\title{
Gender Difference in Arterial Stiffness in a Multicenter Cross-Sectional Study: The Korean Arterial Aging Study (KAAS)
}

\author{
Jang-Young Kim ${ }^{a}$ Jeong Bae Park ${ }^{b}$ Dong Soo Kim ${ }^{d}$ Kee Sik Kim ${ }^{e}$ \\ Jin Won Jeong ${ }^{f}$ Jong Chun Park ${ }^{g}$ Byung Hee Oh ${ }^{\text {h }}$ Namsik Chung ${ }^{c}$ \\ on behalf of the KAAS investigators
}

a Division of Cardiology, Department of Internal Medicine, Yonsei University Wonju Christian Hospital, Wonju, ${ }^{b}$ Department of Internal Medicine, Cheil General Hospital, Kwandong University College of Medicine, and ${ }^{\mathrm{C} C a r d i o l o g y}$ Division, Severance Cardiovascular Hospital, Yonsei University College of Medicine, Seoul, d Department of Internal Medicine, Busan Paik Hospital, University of Inje College of Medicine, Busan, e Department of Internal Medicine, Daegu Catholic University Medical Center, Daegu, ${ }^{f}$ Department of Cardiovascular Medicine, Regional Cardiocerebrovascular Center, Wonkwang University Hospital, Iksan, gDepartment of Internal Medicine, Chonnam National University Medical School, Gwangju, and ${ }^{\mathrm{h}}$ Department of Internal Medicine, Seoul National University College of Medicine, Seongnam, Korea

\section{Key Words}

Age $\cdot$ Arterial stiffness $\cdot$ Gender $\cdot$ Risk factor

\begin{abstract}
Elevated arterial stiffness has emerged as an important risk factor for future cardiovascular (CV) events in men and women. However, gender-related differences in arterial stiffness have not been clearly demonstrated. We thus determine whether gender affects arterial stiffness in subjects with and without CV risk factors. We consecutively enrolled 1,588 subjects aged 17-87 years (mean age: $46.5 ; 51 \%$ women) from the Korean Arterial Aging Study (KAAS), which is a multicenter registry from 13 university hospitals in Korea for the evaluation of arterial stiffness. We compared markers of arterial stiffness - central augmentation index (AIx), aortic pulse wave velocity (PWV), and pulse pressure (PP) amplification - in apparently healthy men and women without risk factors with those in high-risk subjects with a smoking habit, hypertension, diabetes, and dyslipidemia but without drug treatment. Aortic PWV and PP amplification were significantly higher in men than in women $(7.78 \pm 1.16$ vs. $7.64 \pm 1.15 \mathrm{~m} / \mathrm{s}, \mathrm{p}=$ 0.015 , and $1.39 \pm 0.22$ vs. $1.30 \pm 0.18, p<0.001$, respectively). However, women had a significantly higher central AIx than men $(23.5 \pm 11.9$ vs. $16.1 \pm 12.6 \%, p<0.001)$. The central AIx and aortic PWV values were significantly higher in the high-risk group than in the healthy
\end{abstract}


Kim et al.: Gender Difference in Arterial Stiffness in a Multicenter Cross-Sectional Study: The Korean Arterial Aging Study (KAAS)

group for both men and women. In men, central AIx and aortic PWV were associated positively with age and blood pressure, and negatively with body mass index. In women, central AIx was positively related to age, diastolic blood pressure, and serum cholesterol levels. Aortic PWV was positively related to age, systolic blood pressure, fasting glucose, and heart rate. PP amplification was associated negatively with age and blood pressure and positively with heart rate in both men and women. In conclusion, arterial stiffness is mainly determined by sex, age, and blood pressure. Markers of arterial stiffness differ between men and women. Dyslipidemia and glucose contribute to a modest increase in arterial stiffness only in women. Therefore, the arteries of women may be more vulnerable to CV risk factors than those of men.

(C) 2014 S. Karger AG, Basel

\section{Introduction}

Elevated arterial stiffness is recognized as a surrogate end point for cardiovascular (CV) disease because it is closely associated with the presence of CV risk factors, subclinical atherosclerosis, and clinical CV disease including angina, myocardial infarction, stroke, and heart failure. Recent epidemiological and clinical studies have also demonstrated that arterial stiffness measured noninvasively by pulse wave analysis (PWA) and aortic pulse wave velocity (PWV) predicts the risk of total and CV mortality as well as future CV events $[1,2]$.

PWA and aortic PWV measured by arterial applanation tonometry are generally considered the standard methods for evaluating arterial stiffness. PWA allows an estimation of the central augmentation index (AIx) and pulse pressure (PP) amplification. The central AIx is dependent on arterial stiffness and reflective properties, which have been shown to predict CV prognosis [3, 4]. Aortic PWV is a direct measure of arterial stiffness between the carotid and the femoral artery. A high aortic PWV reflects decreased arterial elasticity of a large elastic artery and increased aortic stiffness between two arterial sites [3]. PP amplification is the ratio of carotid to peripheral PP, which is less dependent on blood pressure but an independent risk factor for CV mortality [5].

The central AIx, PP amplification, and aortic PWV are consistently affected by age and blood pressure. However, other risk factors including diabetes, dyslipidemia, and smoking have an inconsistent influence on the three measures [6, 7]. In addition, several studies have shown that arterial stiffness differs considerably between men and women in terms of etiology, pathogenesis, and clinical outcomes because of numerous endogenous factors, such as body size, sex hormones, and biochemical properties of the arteries, and various exogenous factors [8-10].

However, little is known about the determinants of increased arterial stiffness in analyses stratified by gender. We thus studied whether gender affects arterial stiffness differently in subjects with and without CV risk factors, using the Korean Arterial Aging Study (KAAS) data.

\section{Subjects and Methods}

\section{Study Subjects}

We consecutively enrolled 1,588 subjects aged 17-87 years (mean age: 46.5; 51\% women) from the KAAS, which is a multicenter registry from 13 university hospitals in Korea for the evaluation of arterial stiffness. We compared the arterial stiffness indices (central AIx, aortic PWV, and PP amplification) in healthy men and women without risk factors (251 men and 417 women) with those in high-risk subjects with a smoking habit, hypertension, diabetes, and dyslipidemia. Hypertension was defined as blood pressure $\geq 140 / 90 \mathrm{~mm} \mathrm{Hg}$, diabetes as fasting blood glucose $\geq 126 \mathrm{mg} / \mathrm{dl}$ or glycated hemoglobin $\left(\mathrm{HbA}_{1 \mathrm{c}}\right) \geq 6.5 \%$, and 
Kim et al.: Gender Difference in Arterial Stiffness in a Multicenter Cross-Sectional Study: The Korean Arterial Aging Study (KAAS)

dyslipidemia as serum total cholesterol $\geq 240 \mathrm{mg} / \mathrm{dl}$. All subjects were unmedicated. The study protocol was approved by the Institutional Review Boards of the 13 hospitals involved. All participants provided written informed consent.

\section{General Data Collection}

The study participants completed a standardized medical history and lifestyle questionnaire and underwent a comprehensive health examination. Body weight and height were measured while participants were wearing light clothing without shoes. Systolic blood pressure (SBP) and diastolic blood pressure (DBP) were measured twice in the right arm, using an automatic cuff oscillometric device (HEM-705CP; Omron Corp., Kyoto, Japan). The second blood pressure measurement of the two readings was used for data analyses. Smoking status was determined based on self-report. Current smokers were defined as participants who had smoked $\geq 100$ cigarettes in their lifetime and who reported 'currently smoking' in the questionnaire. A venous blood sample was drawn from the study participants after fasting for $>12 \mathrm{~h}$ or overnight.

\section{PWA and PWV by Applanation Tonometry}

The PWA obtained using the GAON 21 System (Hanbyul Meditech, Jeonju, Korea) provides central aortic pressure and the AIx. The latter was calculated from the left radial arterial pulse waves. The data were directly collected in a portable computer, and integral software was used to generate an averaged waveform. The systolic part of the peripheral arterial waveform was characterized by two pressure peaks; the first peak was caused by the left ventricular ejection, whereas the second peak was a result of the wave reflection. The difference between the pressure peaks reflected the degree to which the peripheral arterial pressure was augmented by the wave reflection. The peripheral AIx was defined as the ratio of the second to the first peak of the pressure wave expressed as a percentage. The central AIx was calculated from the peripheral AIx by an automatic mathematical transformation. Differences in consecutive mean data during the repeated measurements were $<0.5 \mathrm{~m} / \mathrm{s}$, and the mean value was used for the final analysis. The peripheral and central PPs were defined as the difference between the SBP and the DBP derived from the brachial blood pressure and aortic pulse wave, respectively. The intra-assay and interassay coefficients of variation of the AIx were 7.1 and $9.1 \%$, respectively.

The PP-1000 system (Hanbyul Meditech) provides aortic PWV values based on the results of simultaneous measurements of pulse waves from arteries at carotid and femoral arterial sites and the time delay $(\Delta t$ or transit time) between the two wave forms. The surface distance (D) between the two recording sites of a pulse wave was measured and entered into the system to allow the calculation of aortic PWV values. PWV was calculated as $\mathrm{PWV}=\mathrm{D} / \Delta \mathrm{t}(\mathrm{m} / \mathrm{s})$. The interobserver difference (mean $\pm 2 \mathrm{SD}$ ) for aortic PWV was $0.14 \pm$ $0.62 \mathrm{~m} / \mathrm{s}$, and the correlation coefficients were significant for it $(\mathrm{r}=0.93)$. The intraobserver difference (mean \pm 2 SD) for aortic PWV was $0.01 \pm 0.03 \mathrm{~m} / \mathrm{s}$, and the correlation coefficients were significant for it as well $(r=0.99)[11]$.

\section{Statistical Analysis}

Unpaired two-sample $t$ tests and $\chi^{2}$ tests were used to examine differences in baseline characteristics and hemodynamic variables including central AIx, aortic PWV, and PP amplification between genders as well as stratified analyses by the presence of risk factors for coronary heart disease. Multivariable linear regression was used to explore the factors determining AIx, aortic PWV, and PP amplification, adjusted for age, body mass index (BMI), smoking, heart rate, blood pressure, fasting glucose, and total cholesterol. All analyses were performed using SPSS version 12.0 (SPSS Inc., USA). p values $<0.05$ were considered to be statistically significant.

\section{Results}

Baseline height, BMI, smoking history, SBP, DBP, fasting glucose, total cholesterol, and serum creatinine were significantly higher in men than in women (table 1). Aortic PWV and PP amplification were significantly higher in men than in women (7.78 \pm 1.16 vs. $7.64 \pm 1.15$ $\mathrm{m} / \mathrm{s}, \mathrm{p}=0.015$, and $1.39 \pm 0.22$ vs. $1.30 \pm 0.18, \mathrm{p}<0.001$, respectively). However, women had a significantly higher central AIx at a heart rate of 75 beats/min than men ( $23.5 \pm 11.9 \mathrm{vs} .16 .1$ $\pm 12.6 \%, \mathrm{p}<0.001$ ). 
Kim et al.: Gender Difference in Arterial Stiffness in a Multicenter Cross-Sectiona Study: The Korean Arterial Aging Study (KAAS)

Table 1. Baseline and hemodynamic characteristics of the study subjects

\begin{tabular}{|c|c|c|c|}
\hline & Men $(\mathrm{n}=778)$ & Women $(\mathrm{n}=810)$ & $\mathrm{p}$ \\
\hline Age, years & $47.32 \pm 11.63$ & $46.19 \pm 13.29$ & 0.072 \\
\hline Height, m & $1.70 \pm 0.06$ & $1.58 \pm 0.06$ & $<0.001$ \\
\hline BMI & $24.57 \pm 2.80$ & $22.86 \pm 3.11$ & $<0.001$ \\
\hline Current smoker, n (\%) & $288(36.46)$ & $28(3.51)$ & $<0.001$ \\
\hline Hypertension, n (\%) & $244(30.89)$ & $138(17.29)$ & $<0.001$ \\
\hline Diabetes, n (\%) & $39(4.94)$ & $25(3.13)$ & 0.067 \\
\hline Dyslipidemia, n (\%) & $228(28.86)$ & $278(34.84)$ & 0.010 \\
\hline Total cholesterol, mg/dl & $191.57 \pm 34.90$ & $188.46 \pm 35.82$ & 0.079 \\
\hline Fasting glucose, mg/dl & $98.22 \pm 19.14$ & $91.97 \pm 15.63$ & $<0.001$ \\
\hline Serum creatinine, $\mathrm{mg} / \mathrm{dl}$ & $1.00 \pm 0.17$ & $0.74 \pm 0.15$ & $<0.001$ \\
\hline Heart rate, beats/min & $67.01 \pm 10.30$ & $66.80 \pm 9.56$ & 0.700 \\
\hline \multicolumn{4}{|l|}{$\mathrm{SBP}, \mathrm{mm} \mathrm{Hg}$} \\
\hline Peripheral & $129.04 \pm 16.83$ & $120.23 \pm 18.58$ & $<0.001$ \\
\hline Central & $117.19 \pm 16.57$ & $111.66 \pm 18.72$ & $<0.001$ \\
\hline \multicolumn{4}{|l|}{$\mathrm{DBP}, \mathrm{mm} \mathrm{Hg}$} \\
\hline Peripheral & $80.48 \pm 12.30$ & $74.64 \pm 11.58$ & $<0.001$ \\
\hline Central & $81.59 \pm 12.34$ & $75.81 \pm 11.73$ & $<0.001$ \\
\hline \multicolumn{4}{|l|}{$\mathrm{PP}, \mathrm{mm} \mathrm{Hg}$} \\
\hline Peripheral & $48.56 \pm 10.59$ & $45.58 \pm 11.63$ & $<0.001$ \\
\hline Central & $35.60 \pm 9.04$ & $35.86 \pm 10.74$ & 0.609 \\
\hline AIx, \% & $19.44 \pm 13.03$ & $26.88 \pm 12.34$ & $<0.001$ \\
\hline AIx at a heart rate of 75 beats $/ \mathrm{min}, \%$ & $16.14 \pm 12.60$ & $23.49 \pm 11.85$ & $<0.001$ \\
\hline \multicolumn{4}{|l|}{$\mathrm{PWV}, \mathrm{m} / \mathrm{s}$} \\
\hline Aortic & $7.78 \pm 1.16$ & $7.64 \pm 1.15$ & 0.015 \\
\hline Leg & $10.09 \pm 1.36$ & $9.49 \pm 1.30$ & $<0.001$ \\
\hline Arm & $8.79 \pm 1.35$ & $8.36 \pm 1.22$ & $<0.001$ \\
\hline PP amplification & $1.39 \pm 0.22$ & $1.30 \pm 0.18$ & $<0.001$ \\
\hline
\end{tabular}

The central AIx and aortic PWV values were significantly higher in the subgroup with hypertension, diabetes, and dyslipidemia than in the no-risk group for both men and women (table 2). However, smoking did not influence any of the three markers. PP amplification was significantly lower in the female subgroup with hypertension and dyslipidemia than in the male subgroup.

\section{Multiple Linear Regression Analysis of the Risk Factors regarding Their Influence on}

Arterial Stiffness Indices

Multiple linear regression analyses revealed that women had significantly higher central AIx $(\beta=4.94, p<0.001)$ and lower PP amplification values $(\beta=-0.11, p<0.001)$ than men. There were differences in the associations of risk factors with the central AIx at a heart rate of 75 beats/min, aortic PWV, and PP amplification between men and women (table 3).

In men, the central AIx at a heart rate of 75 beats/min and aortic PWV were associated positively with age and blood pressure and negatively with BMI. DBP was more strongly related to central AIx, and SBP was more strongly related to aortic PWV. PP amplification was associated negatively with age and blood pressure and positively with heart rate.

In women, the central AIx at a heart rate of 75 beats/min was associated positively with age, DBP, and serum cholesterol levels. Aortic PWV was positively related to age, SBP, fasting glucose, and heart rate. PP amplification was associated negatively with age and blood pressure and positively with heart rate. 
Kim et al.: Gender Difference in Arterial Stiffness in a Multicenter Cross-Sectional Study: The Korean Arterial Aging Study (KAAS)
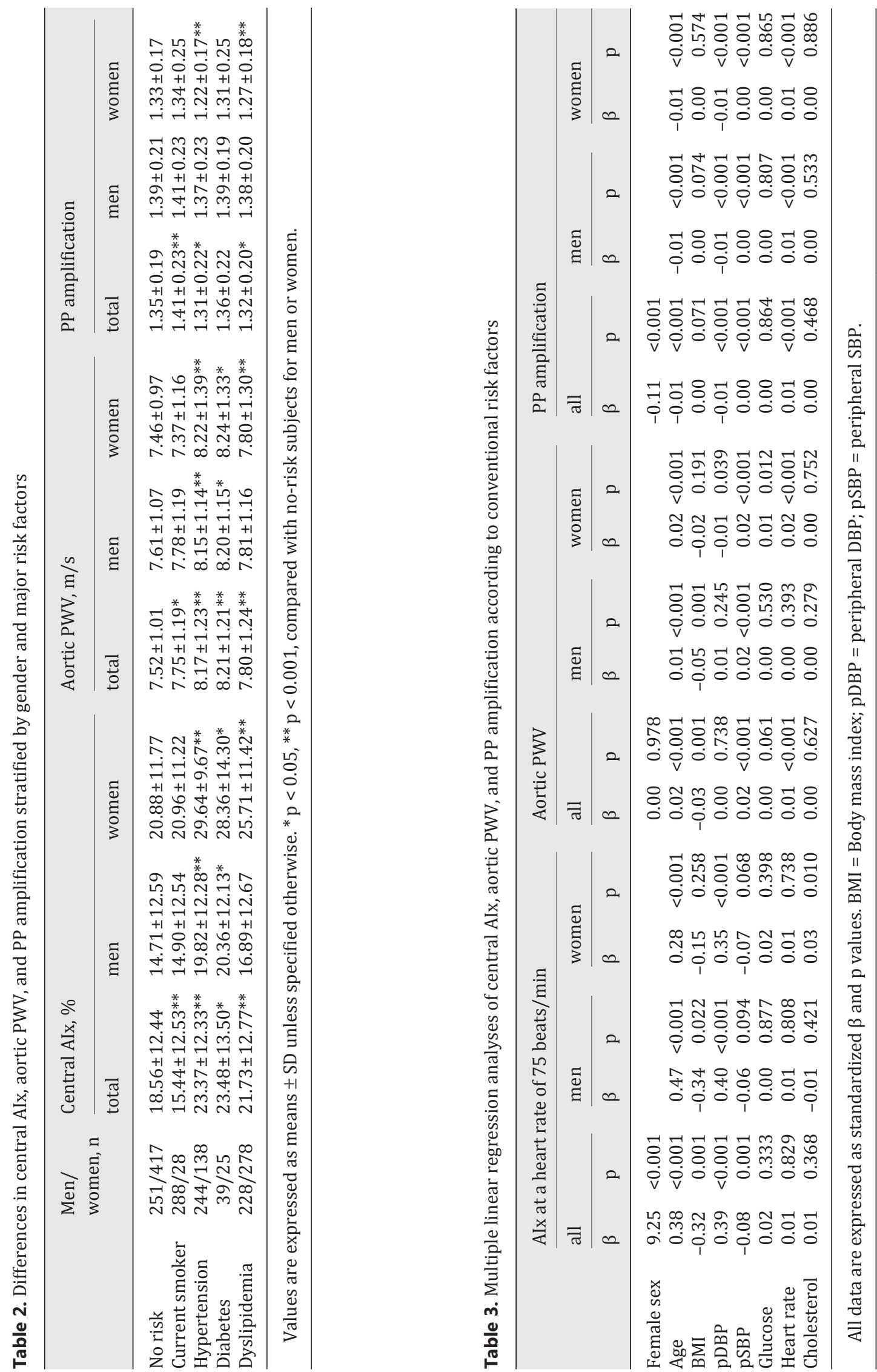
Kim et al.: Gender Difference in Arterial Stiffness in a Multicenter Cross-Sectional Study: The Korean Arterial Aging Study (KAAS)

\section{Discussion}

In this multicenter registry from 13 Korean university hospitals for the evaluation of arterial stiffness, a sex-related difference was observed for central AIx but not for aortic PWV. Age and blood pressure were strongly associated with central AIx and aortic PWV in both men and women. DBP was more strongly related to central AIx, and SBP was more strongly associated with aortic PWV. BMI affected both the central AIx and aortic PWV in men but not in women. The total cholesterol level and fasting blood glucose contributed to the central AIx and aortic PWV only in women.

The central AIx was consistently higher in women than in men irrespective of the number of risk factors. The shorter average height of women than of men (158.3 vs. $170.1 \mathrm{~cm}$ in this study) results in an earlier wave reflection to the central aorta in systole than in diastole in women because of a closer proximity between the heart and the sites of wave reflection [8, 12-14]. Height is also directly related to arterial caliber and length as well as cardiac index, which contribute to a higher central AIx in woman. However, even when corrected for BMI or height, the central AIx remained significantly higher in women and gender remained independently associated with central AIx in our multiple regression analyses. These results suggest that our observation of a higher central AIx in women is partly due to gender differences in wave reflection rather than an effect on large artery stiffness or short status. Sex steroids such as estrogen and progesterone could influence the gender difference in arterial stiffness. However, we could not confirm these associations in the KAAS because we did not measure sex hormones and menopausal status in women. This issue requires further investigation $[8,14]$.

Age, heart rate, and blood pressure were key determinants of the central AIx and aortic PWV in both men and women in this study, which is consistent with results of previous studies $[3,10,15]$. Interestingly, BMI was associated with aortic PWV and AIx only in men, whereas total cholesterol and fasting blood glucose were associated with aortic PWV and AIx only in women. The influence of gender on the impact of metabolic risk factors on aortic PWV and the central AIx is poorly described $[16,17]$. It is possible that high cholesterol levels, hyperglycemia, and renal dysfunction may interact with estrogen or progesterone to modify the biochemical elastic properties of the arterial wall, which might, in turn, influence pressure wave reflections as well as large artery stiffness [14].

Some limitations of our study should also be considered. First, the study was restricted to middle-aged and elderly subjects visiting university hospitals, which cannot represent a healthy adult Korean population. Second, it was a cross-sectional study; therefore, determining cause-effect interferences was difficult. Third, we did not investigate any family history of CV disease. Thus, we could not evaluate the relationship between family history and risk factors as well as arterial stiffness. Finally, our analyses were based on a single determination of the central AIx and aortic PWV, which is subject to random measurement error and may have underestimated the strength of the associations.

\section{Acknowledgements}

The study was sponsored by Boryung Pharmaceutical and supported by the KAAS investigators. The KAAS investigators are Professors Dong Soo Kim, Jin Won Jeong, Shung Chull Chae, Jong Chun Park, Jang Ho Bae, Myeong Chan Cho, Ho Joong Youn, Sang Hong Baek, Chang Gyu Park, Su-Yeon Choi, Kyu-Hyung Ryu, Dong-Ju Choi, Moo-Yong Rhee, Sungha Park, Cheol-Ho Kim, Se-Joong Rim, Dong-Gu Shin, Kye Hun Kim, Won Ho Kim, Wang Seong Ryu, Kyeong Ho Yun, Young Moo Ro, and Young-Dea Kim. 
Kim et al.: Gender Difference in Arterial Stiffness in a Multicenter Cross-Sectional Study: The Korean Arterial Aging Study (KAAS)

\section{References}

1 Vlachopoulos C, Aznaouridis K, Stefanadis C: Prediction of cardiovascular events and all-cause mortality with arterial stiffness: a systematic review and meta-analysis. J Am Coll Cardiol 2010;55:1318-1327.

-2 Wang KL, Cheng HM, Sung SH, Chuan SY, Li CH, Spurgeon HA, Ting CT, Najjar SS, Lakatta EG, Yin FC, Chou P, Chen $\mathrm{CH}$ : Wave reflection and arterial stiffness in the prediction of 15-year all-cause and cardiovascular mortalities: a community-based study. Hypertension 2010;55:799-805.

-3 Laurent S, Cockcroft J, van Bortel L, Boutouyrie P, Giannattasio C, Hayoz D, Pannier B, Vlachopoulos C, Wilkinson I, Struijker-Boudier H; European Network for Non-Invasive Investigation of Large Arteries: Expert consensus document on arterial stiffness: methodological issues and clinical applications. Eur Heart J 2006; 27:2588-2605.

-4 Williams B, Lacy PS, Thom SM, Cruickshank K, Stanton A, Collier D, Hughes AD, Thurston H, O’Rourke M; CAFE Investigators; Anglo-Scandinavian Cardiac Outcomes Trial Investigators; CAFE Steering Committee and Writing Committee: Differential impact of blood pressure-lowering drugs on central aortic pressure and clinical outcomes: principal results of the Conduit Artery Function Evaluation (CAFE) study. Circulation 2006; 113:1213-1225.

5 Benetos A, Thomas F, Joly L, Blacher J, Pannier B, Labat C, Salvi P, Smulyan H, Safar ME: Pulse pressure amplification: a mechanical biomarker of cardiovascular risk. J Am Coll Cardiol 2010;55:1032-1037.

6 Yasmin, Wallace S, McEniery CM, Dakham Z, Pusalkar P, Maki-Petaja K, Ashby MJ, Cockcroft JR, Wilkinson IB: Matrix metalloproteinase-9 (MMP-9), MMP-2, and serum elastase activity are associated with systolic hypertension and arterial stiffness. Arterioscler Thromb Vasc Biol 2005;25:372.

-7 McEniery CM, Yasmin, Maki-Petaja KM, McDonnell BJ, Munnery M, Hickson SS, Franklin SS, Cockcroft JR, Wilkinson IB; Anglo-Cardiff Collaboration Trial Investigators: The impact of cardiovascular risk factors on aortic stiffness and wave reflections depends on age: the Anglo-Cardiff Collaborative Trial (ACCT III). Hypertension 2010;56:591-597.

-8 Rossi P, Frances Y, Kingwell BA, Ahimastos AA: Gender differences in artery wall biomechanical properties throughout life. J Hypertens 2011;29:1023-1033.

-9 Segers P, Rietzschel ER, de Buyzere ML, Vermeersch SJ, de Bacquer D, van Bortel LM, de Backer G, Gillebert TC, Verdonck PR; Asklepios Investigators: Noninvasive (input) impedance, pulse wave velocity, and wave reflection in healthy middle-aged men and women. Hypertension 2007;49:1248-1255.

10 Janner JH, Godtfredsen NS, Ladelund S, Vestbo J, Prescott E: Aortic augmentation index: reference values in a large unselected population by means of the SphygmoCor device. Am J Hypertens 2010;23:180-185.

$\checkmark 11$ Lee NB, Park CG: Reproducibility of regional pulse wave velocity in healthy subjects. Korean J Intern Med 2009;24:19-23.

-12 Smulyan H, Marchais SJ, Pannier B, Guerin AP, Safar ME, London GM: Influence of body height on pulsatile arterial hemodynamic data. J Am Coll Cardiol 1998;31:1103-1109.

-13 McGrath BP, Liang YL, Kotsopoulos D, Cameron JD: Impact of physical and physiological factors on arterial function. Clin Exp Pharmacol Physiol 2001;28:1104-1107.

14 Vitale C, Mendelsohn ME, Rosano GM: Gender differences in the cardiovascular effect of sex hormones. Nat Rev Cardiol 2009;6:532-542.

15 McEniery CM, Yasmin, Hall IR, Qasem A, Wilkinson IB, Cockcroft JR; ACCT Investigators: Normal vascular aging: differential effects on wave reflection and aortic pulse wave velocity: the Anglo-Cardiff Collaborative Trial (ACCT). J Am Coll Cardiol 2005;46:1753-1760.

-16 Protogerou AD, Blacher J, Aslangul E, le Jeunne C, Lekakis J, Mavrikakis M, Safar ME: Gender influence on metabolic syndrome's effects on arterial stiffness and pressure wave reflections in treated hypertensive subjects. Atherosclerosis 2007;193:151-158.

17 Aoun S, Blacher J, Safar ME, Mourad JJ: Diabetes mellitus and renal failure: effects on large artery stiffness. J Hum Hypertens 2001;15:693-700. 